\title{
WEAK DISTRIBUTIVITY IMPLYING DISTRIBUTIVITY
}

\author{
DAN HATHAWAY
}

\begin{abstract}
Let $\mathbb{B}$ be a complete Boolean algebra. We show that if $\lambda$ is an infinite cardinal and $\mathbb{B}$ is weakly $\left(\lambda^{\omega}, \omega\right)$-distributive, then $\mathbb{B}$ is $(\lambda, 2)$-distributive. Using a similar argument, we show that if $\kappa$ is a weakly compact cardinal such that $\mathbb{B}$ is weakly $\left(2^{\kappa}, \kappa\right)$ distributive and $\mathbb{B}$ is $(\alpha, 2)$-distributive for each $\alpha<\kappa$, then $\mathbb{B}$ is $(\kappa, 2)$-distributive.
\end{abstract}

\section{INTRODUCTION}

Given sets $A$ and $B,{ }^{A} B$ denotes the set of functions from $A$ to $B$. In this article, $\lambda$ and $\kappa$ will denote ordinals, although usually they can be assumed to be infinite cardinals. As defined in [6], given $\lambda$ and $\kappa$, we say that a complete Boolean algebra $\mathbb{B}$ is $(\lambda, \kappa)$-distributive iff

$$
\prod_{\alpha<\lambda} \sum_{\beta<\kappa} u_{\alpha, \beta}=\sum_{f: \lambda \rightarrow \kappa} \prod_{\alpha<\lambda} u_{\alpha, f(\alpha)}
$$

for any $\left\langle u_{\alpha, \beta} \in \mathbb{B}: \alpha<\lambda, \beta<\kappa\right\rangle$. Given maximal antichains $A_{1}, A_{2} \subseteq$ $\mathbb{B}$, we say that $A_{2}$ refines $A_{1}$ iff $\left(\forall a_{2} \in A_{2}\right)\left(\exists a_{1} \in A_{1}\right) a_{2} \leq_{\mathbb{B}} a_{1}$. It is a fact that $\mathbb{B}$ is $(\lambda, \kappa)$-distributive iff each size $\lambda$ collection of maximal antichains in $\mathbb{B}$ each of size $\kappa$ has a common refinement. There is also a useful characterization in terms of forcing (which can be found in [6] as Theorem 15.38):

Fact 1.1. A complete Boolean algebra $\mathbb{B}$ is $(\lambda, \kappa)$-distributive iff

$$
1 \Vdash_{\mathbb{B}}(\forall f: \check{\lambda} \rightarrow \check{\kappa}) f \in \check{V} .
$$

Unfortunately, the definition of weakly distributive varies in the literature (for example [7]). We will use the one given by Jech (see [6]). That is, we say that a complete Boolean algebra $\mathbb{B}$ is weakly $(\lambda, \kappa)$ distributive iff

$$
\prod_{\alpha<\lambda} \sum_{\beta<\kappa} u_{\alpha, \beta}=\sum_{g: \lambda \rightarrow \kappa} \prod_{\alpha<\lambda} \sum_{\beta<g(\alpha)} u_{\alpha, \beta} .
$$

A portion of the results of this paper were proven during the September 2012 Fields Institute Workshop on Forcing while the author was supported by the Fields Institute. Work was also done wile under NSF grant DMS-0943832. 
This definition has a natural characterization in terms of forcing. Given a set $X$ and $f, g: X \rightarrow \kappa$, we write $f \leq g$ iff $g$ everywhere dominates $f$. That is,

$$
(\forall x \in X) f(x) \leq g(x) .
$$

Fact 1.2. A complete Boolean algebra $\mathbb{B}$ is weakly $(\lambda, \kappa)$-distributive iff

$$
1 \Vdash_{\mathbb{B}}(\forall f: \check{\lambda} \rightarrow \check{\kappa})(\exists g: \check{\lambda} \rightarrow \check{\kappa}) g \in \check{V} \wedge f \leq g .
$$

We will show the following:

Theorem (A). Let $\lambda$ be an infinite cardinal. If

1) $\mathbb{B}$ is weakly $\left(\lambda^{\omega}, \omega\right)$-distributive, then $\mathbb{B}$ is $(\lambda, 2)$-distributive.

Theorem (B). Let $\kappa$ be a weakly compact cardinal. If

1) $\mathbb{B}$ is weakly $\left(2^{\kappa}, \kappa\right)$-distributive and

2) $\mathbb{B}$ is $(\alpha, 2)$-distributive for each $\alpha<\kappa$,

then $\mathbb{B}$ is $(\kappa, 2)$-distributive.

We will then discuss why Theorem (B) does not hold when we have $\kappa=\omega_{1}$ instead of $\kappa$ being weakly compact, and we will show one way to fix the situation using the tower number. Finally, we use the same idea using the tower number to prove a variation of Theorem (A) involving weak $\left(\lambda^{\kappa}, \kappa\right)$-distributivity for $\kappa>\omega$.

\section{Functions From $\lambda^{\omega}$ TO $\omega$}

The proof of the following lemma uses the fact that well-foundedness of trees is absolute. It is crucial, for what follows, that this lemma does not require ${ }^{\omega} \lambda \subseteq M$. See [4] for motivation and discussion.

Lemma 2.1. For each $A \subseteq \lambda$, there is a function $f:{ }^{\omega} \lambda \rightarrow \omega$ such that whenever $M$ is a transitive model of $Z F$ such that $\lambda \in M$ and some $g:\left({ }^{\omega} \lambda\right)^{M} \rightarrow \omega$ in $M$ satisfies

$$
\left(\forall x \in\left({ }^{\omega} \lambda\right)^{M}\right) f(x) \leq g(x),
$$

then $A \in M$.

Proof. Fix $A \subseteq \lambda$. Define $f:{ }^{\omega} \lambda \rightarrow \omega$ by

$$
f(x):= \begin{cases}0 & \text { if }(\forall n<\omega) x(n) \notin A, \\ n+1 & \text { if } x(n) \in A \text { but }(\forall m<n) x(m) \notin A .\end{cases}
$$


Let $M$ be a transitive model of ZF such that $\lambda \in M$ but $A \notin M$. Suppose, towards a contradiction, that there is some $g \in M$ satisfying $\left(\forall x \in\left({ }^{\omega} \lambda\right)^{M}\right) f(x) \leq g(x)$. Let $B$ be the set

$$
B:=\left\{t \in{ }^{<\omega} \lambda: g(x) \geq|t| \text { for all } x \text { in } M \text { extending } t\right\} .
$$

Notice that $B \in M$. Let $T \subseteq{ }^{<\omega} \lambda$ be the set of elements of $B$ all of whose initial segments are also in $B$. Note that $T$ is a tree and $T \in M$.

For all $a \in \lambda, a \in A$ implies $\langle a\rangle \in B$. Thus, there must be some $a_{0} \in \lambda$ such that $a_{0} \notin A$ but $\left\langle a_{0}\right\rangle \in B$. If there was not, then $A$ could be defined in $M$ by $A=\{a \in \lambda:\langle a\rangle \in B\}$.

Next, for all $a \in \lambda, a \in A$ implies $\left\langle a_{0}, a\right\rangle \in B$. Thus, by similar reasoning as before, there must be some $a_{1} \in \lambda$ such that $a_{1} \notin A$ but $\left\langle a_{0}, a_{1}\right\rangle \in B$. Continuing like this, we can construct a sequence $x \in{ }^{\omega} \lambda$ satisfying $(\forall n<\omega) x \uparrow n \in B$. Thus, $(\forall n<\omega) x \uparrow n \in T$, so $T$ is not well-founded.

Since well-foundedness is absolute, there is some path $x^{\prime}$ through $T$ in $M$. Since $(\forall n<\omega) x^{\prime} \uparrow n \in B$, we have $(\forall n<\omega) g\left(x^{\prime}\right) \geq n$, which is impossible.

This implies the following lemma, whose order of quantifiers is not as powerful, but the functions have the ordinal $\left(\lambda^{\omega}\right)^{M}$ instead of the set of sequences $\left({ }^{\omega} \lambda\right)^{M}$ as their domains:

Lemma 2.2. Let $M$ be a transitive model of $Z F$ such that the ordinal $\lambda$ is in $M$ and $\left({ }^{\omega} \lambda\right)^{M}$ can be well-ordered in $M$. Assume that for each $f:\left(\lambda^{\omega}\right)^{M} \rightarrow \omega$ there is some $g:\left(\lambda^{\omega}\right)^{M} \rightarrow \omega$ in $M$ such that $f \leq g$. Then $\mathcal{P}(\lambda) \subseteq M$.

Proof. Consider any $A \in \mathcal{P}(\lambda)$. Use the lemma above with $A$ to get $\tilde{f}:{ }^{\omega} \lambda \rightarrow \omega$ such that if $\tilde{g}:\left({ }^{\omega} \lambda\right)^{M} \rightarrow \omega$ is any function in $M$ which satisfies

$$
\left(\forall x \in\left({ }^{\omega} \lambda\right)^{M}\right) \tilde{f}(x) \leq \tilde{g}(x)
$$

then $A \in M$. Since $\left({ }^{\omega} \lambda\right)^{M}$ can be well-ordered in $M$, fix a bijection

$$
\eta:\left(\lambda^{\omega}\right)^{M} \rightarrow\left({ }^{\omega} \lambda\right)^{M}
$$

in $M$. Define $f:\left(\lambda^{\omega}\right)^{M} \rightarrow \omega$ by

$$
f(\alpha):=\tilde{f}(\eta(\alpha)) .
$$


That is, the following diagram commutes:

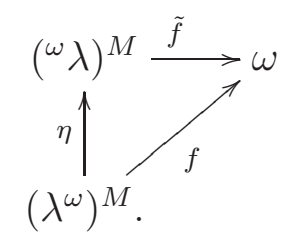

By hypothesis, let $g:\left(\lambda^{\omega}\right)^{M} \rightarrow \omega$ be a function in $M$ which everywhere dominates $f$. Define $\tilde{g}:\left({ }^{\omega} \lambda\right)^{M} \rightarrow \omega$ by

$$
\tilde{g}(x):=g\left(\eta^{-1}(x)\right) \text {. }
$$

We have that $\tilde{g} \in M$ and $\tilde{g}$ satisfies 1, so by the hypothesis on $\tilde{f}$, $A \in M$.

We now have the main result of this section:

Theorem (A). Let $\mathbb{B}$ be a complete Boolean algebra and $\lambda$ be an infinite cardinal. If $\mathbb{B}$ is weakly $\left(\lambda^{\omega}, \omega\right)$-distributive, then $\mathbb{B}$ is $(\lambda, 2)$ distributive.

Proof. Let $\mu:=\lambda^{\omega}$. Assume $\mathbb{B}$ is weakly $(\mu, \omega)$-distributive. Force with $\mathbb{B}$. Every $f: \mu \rightarrow \omega$ in the extension can be everywhere dominated by some $g: \mu \rightarrow \omega$ in the ground model, so applying the lemma above in the extension (setting $M$ to be the ground model) tells us that the $\mathcal{P}(\lambda)$ of the extension is included in the ground model. Hence, $\mathbb{B}$ is $(\lambda, 2)$-distributive.

\section{Functions from $2^{\kappa}$ TO $\kappa$ With $\kappa$ Weakly Compact}

The first lemma in the previous section was the key to the theorem there. We have a parallel lemma here which, instead of using the absoluteness of trees being well-founded, uses the tree property to get similar absoluteness. It is important that this lemma does not require ${ }^{\kappa_{2}} \subseteq M$. By weakly compact, we mean strongly inaccessible and having the tree property.

Lemma 3.1. For each $a \in{ }^{\kappa} 2$, there is a function $f:{ }^{\kappa} 2 \rightarrow \kappa$ such that whenever $M$ is a transitive model of $Z F$ such that $\kappa \in M,<\kappa 2 \subseteq M$, $\left({ }^{\kappa} 2\right)^{M}$ can be well-ordered in $M,(\kappa \text { is weakly compact })^{M}$, and some $g:\left({ }^{\kappa} 2\right)^{M} \rightarrow \kappa$ in $M$ satisfies

$$
\left(\forall x \in\left({ }^{\kappa} 2\right)^{M}\right) f(x) \leq g(x)
$$

then $a \in M$. 
Proof. Fix $a \in{ }^{\kappa} 2$. Let $f:{ }^{\kappa} 2 \rightarrow \kappa$ be the function

$$
f(x):= \begin{cases}0 & \text { if }(\forall \alpha<\kappa) x(\alpha)=a(\alpha), \\ \alpha+1 & \text { if } x(\alpha) \neq a(\alpha) \text { but }(\forall \beta<\alpha) x(\beta)=a(\beta) .\end{cases}
$$

Let $M$ be an appropriate transitive model of ZF. Suppose $g:\left({ }^{\kappa} 2\right)^{M} \rightarrow$ $\kappa$ in $M$ satisfies $\left(\forall x \in\left({ }^{\kappa} 2\right)^{M}\right) f(x) \leq g(x)$. We will show that $a \in M$.

Suppose, towards a contradiction, that $a \notin M$. Let

$$
B:=\left\{t \in{ }^{<\kappa} 2: g(x) \geq \operatorname{Dom}(t) \text { for all } x \text { in } M \text { extending } \mathrm{t}\right\} .
$$

Note that by definition, there cannot be any $x \in{ }^{\kappa} 2$ in $M$ satisfying $(\forall \alpha<\kappa) x \uparrow \alpha \in B$ because if there was such an $x$, we would have $(\forall \alpha<\kappa) g(x) \geq \alpha$, which is impossible. Since $B$ need not be a tree, let $T \subseteq{ }^{<\kappa} 2$ be the tree of those elements of $B$ all of whose initial segments are also in $B$. Again, $T$ cannot have a length $\kappa$ path in $M$. Note that for each $\alpha<\kappa, a \uparrow \alpha \in B$. This is because any $x \in{ }^{\kappa} 2$ in $M$ which extends $a \uparrow \alpha$ differs from $a$ (since $a \notin M$ ), and the smallest $\gamma$ such that $x(\gamma) \neq a(\gamma)$ must be $\geq \alpha$, so

$$
g(x) \geq f(x)=\gamma+1>\gamma \geq \alpha=\operatorname{Dom}(a\lceil\alpha) .
$$

Since $(\forall \alpha<\kappa) a \uparrow \alpha \in B$, also $(\forall \alpha<\kappa) a \uparrow \alpha \in T$.

Now, $B \in M$ (since ${ }^{<\kappa} 2 \subseteq M$ and $g \in M$ ) and so $T \in M$. Since $(\forall \alpha<\kappa) a \uparrow \alpha \in T$, $(T \text { has height } \kappa)^{M}$. Since $(\kappa$ is strongly inaccessible $)^{M}$, we have $(T \text { is a } \kappa \text {-tree })^{M}$. Since $(\kappa \text { has the tree property })^{M}$, there is a length $\kappa$ path through $T$ in $M$, which we said earlier was impossible.

As before, this implies the following lemma, whose order of quantifiers is not as powerful, but the functions have the ordinal $\left(2^{\kappa}\right)^{M}$ instead of the set of sequences $\left({ }^{\kappa} 2\right)^{M}$ as their domains:

Lemma 3.2. Let $M$ be a transitive model of $Z F$ such that the ordinal $\kappa$ is in $M,{ }^{<\kappa} 2 \subseteq M,\left({ }^{\kappa} 2\right)^{M}$ can be well-ordered in $M$, and $(\kappa$ is weakly compact $)^{M}$. Assume that for each $f:\left(2^{\kappa}\right)^{M} \rightarrow \kappa$ there is some $g:\left(2^{\kappa}\right)^{M} \rightarrow \kappa$ in $M$ such that $f \leq g$. Then $\mathcal{P}(\kappa) \subseteq M$.

Proof. The proof is similar to that of Lemma 2.2 .

As before, the main result of this section follows:

Theorem (B). Let $\mathbb{B}$ be a complete Boolean algebra and $\kappa$ be a weakly compact cardinal. If $\mathbb{B}$ is weakly $\left(2^{\kappa}, \kappa\right)$-distributive and $\mathbb{B}$ is $(\alpha, 2)$ distributive for each $\alpha<\kappa$, then $\mathbb{B}$ is $(\kappa, 2)$-distributive.

Proof. This follows from the lemma above just as Theorem (A) followed from Lemma 2.2. 


\section{The Tower Number}

One might hope that Theorem (B) holds when $\kappa=\omega_{1}$ instead of $\kappa$ being weakly compact. That is, one might hope that if a complete Boolean algebra $\mathbb{B}$ is weakly $\left(2^{\omega_{1}}, \omega_{1}\right)$-distributive and $(\omega, 2)$ distributive, then it is $\left(\omega_{1}, 2\right)$-distributive. Unfortunately, this cannot be proved in ZFC because $\mathbb{B}$ could be a Suslin algebra (a Suslin algebra is c.c.c. and therefore is weakly $\left(\lambda, \omega_{1}\right)$-distributive for any $\left.\lambda\right)$. However, if we add the assumption that $1 \Vdash_{\mathbb{B}}\left(\omega_{1}<\mathfrak{t}\right)$, where we will define $\mathfrak{t}$ soon, then $\mathbb{B}$ is $\left(\omega_{1}, 2\right)$-distributive. The argument is simpler than that of Theorem (A) and Theorem(B) and does not need the hypothesis of weak $\left(2^{\omega_{1}}, \omega_{1}\right)$-distributivity. As a final twist, we will combine several ideas to prove a variation of Theorem (A).

Recall that $\mathfrak{t}$, the tower number, is the smallest length of a sequence

$$
\left\langle S_{\alpha} \in[\omega]^{\omega}: \alpha<\kappa\right\rangle
$$

satisfying $(\forall \alpha<\beta<\kappa) S_{\alpha} \supseteq^{*} S_{\beta}$ but there is no $S \in[\omega]^{\omega}$ satisfying $(\forall \alpha<\kappa) S_{\alpha} \supseteq^{*} S$ (where $S_{1} \subseteq^{*} S_{2}$ means $S_{1}-S_{2}$ is finite). It is not hard to see that $\omega_{1} \leq \mathfrak{t} \leq 2^{\omega}$. See [1] for more on $\mathfrak{t}$ and related cardinals. The following lemma is the key. The idea is borrowed from Farah in [3], who got the idea from Dordal in [2], who got the idea from Booth.

Lemma 4.1. Let $\kappa$ be such that $\omega_{1} \leq \kappa<\mathfrak{t}$. Let $M$ be a transitive model of ZFC such that $\kappa \in M$ and $(\forall \alpha<\kappa) \mathcal{P}(\alpha) \subseteq M$. Then $\mathcal{P}(\kappa) \subseteq M$.

Proof. Fix $\kappa$ and $M$. Since $\kappa \in M$ and $(\forall \alpha<\kappa) \mathcal{P}(\alpha) \subseteq M$, we have ${ }^{<\kappa_{2}} \subseteq M$. Let $F:{ }^{<\kappa} 2 \rightarrow[\omega]^{\omega}$ be a function in $M$ such that for all $t_{1}, t_{2} \in{ }^{<\kappa} 2$,

1) $t_{1} \sqsubseteq t_{2} \Rightarrow F\left(t_{1}\right) \supseteq^{*} F\left(t_{2}\right)$, and

2) $t_{1} \perp t_{2} \Rightarrow F\left(t_{1}\right) \cap F\left(t_{2}\right)$ is finite.

Such functions are easy to construct by induction (and the Axoim of Choice). The construction will not get stuck at a limit stage $\gamma<\kappa$ because given $t \in{ }^{\gamma} 2 \subseteq M$ and $\langle F(t \uparrow \alpha): \alpha<\gamma\rangle$, since $\gamma<\mathfrak{t}$ there is some $S \in[\omega]^{\omega} \subseteq M$ such that $(\forall \alpha<\gamma) S \subseteq^{*} F(t\lceil\alpha)$. The set $F(t)$ can be defined to be the least such $S$ accoding to some fixed well-ordering of $[\omega]^{\omega}$.

Now, consider any $a \in{ }^{\kappa} 2$. We will show that $a \in M$. The sequence $\left\langle F(a\lceil\alpha): \alpha<\kappa\rangle\right.$ is a $\supseteq^{*}$-chain (in V) of length $\kappa$. Since $\kappa<\mathfrak{t}$, fix some $S \in[\omega]^{\omega}$ satisfying

$$
(\forall \alpha<\kappa) S \subseteq^{*} F(a \uparrow \alpha) .
$$


Since $\mathcal{P}(\omega) \subseteq M$, in particular $S \in M$. Within $M$, the function $F$ and the set $S$ can be used together to define $a$ :

$$
a=\bigcup\left\{t \in{ }^{<\kappa} 2: S \subseteq^{*} F(t)\right\} .
$$

By applying the lemma above inductively, we get an improvement:

Lemma 4.2. Let $\kappa$ be such that $\omega_{1} \leq \kappa<\mathfrak{t}$. Let $M$ be a transitive model of $Z F C$ such that $\mathcal{P}(\omega) \subseteq M$. Then $\mathcal{P}(\kappa) \subseteq M$.

This last lemma is closely related to the fact that $2^{\kappa}=2^{\omega}$ whenever $\kappa<\mathfrak{t}$. A proof of this using an argument similar to Lemma 4.1 can be found in [1]. Martin's Axiom (MA) implies $\mathfrak{t}=2^{\omega}$, but the original proof [8] that MA implies $2^{\kappa}=2^{\omega}$ whenever $\kappa<2^{\omega}$ used the almost disjoint coding poset. We now have the application to complete Boolean algebras:

Proposition 4.3. Let $\kappa$ be an infinite cardinal. Let $\mathbb{B}$ be a complete Boolean algebra such that $\mathbb{B}$ is $(\omega, 2)$-distributive and $1 \Vdash_{\mathbb{B}}(\check{\kappa}<\mathfrak{t})$. Then $\mathbb{B}$ is $(\kappa, 2)$-distributive.

Proof. Apply Lemma 4.2 in the forcing extension with $M$ equal to the ground model.

Let $\kappa$ be such that $\omega_{1} \leq \kappa<\mathfrak{t}$. Any $A \in[\omega]^{\omega}$ can be partitioned into $2^{\omega}$ infinite sets with pairwise finite intersection. Thus, fixing $\lambda \leq 2^{\omega}$, the function $F:{ }^{<\kappa} 2 \rightarrow[\omega]^{\omega}$ in Lemma 4.1 can be replaced by a function $F:{ }^{<\kappa} \lambda \rightarrow[\omega]^{\omega}$ satisfying the same conditions. Slightly modifying the proof of Lemma 4.1, we get that if $M$ is a transitive model of ZFC such that $\lambda \in M$ and $(\forall \alpha<\kappa)^{\alpha} \lambda \subseteq M$, then ${ }^{\kappa} \lambda \subseteq M$. Inductively applying this fact yields an improvement:

Lemma 4.4. Let $\kappa$ and $\lambda$ be such that $\omega_{1} \leq \kappa<\mathfrak{t}$ and $\lambda \leq 2^{\omega}$. Let $M$ be a transitive model of $Z F C$ such that $\lambda \in M$ and ${ }^{\omega} \lambda \subseteq M$. Then ${ }^{\kappa} \lambda \subseteq M$.

Now we may combine Lemma 4.4 with the argument in Lemma 2.1. The case $\kappa=\omega$ of this next lemma is already handled by Lemma 2.1.

Lemma 4.5. Let $\kappa$ and $\lambda$ be such that $\omega \leq \kappa<\mathfrak{t}$ and $\lambda \leq 2^{\omega}$. For each $A \subseteq \lambda$, there is a function $f:{ }^{\kappa} \lambda \rightarrow \kappa$ such that whenever $M$ is a transitive model of $Z F C$ such that ${ }^{\omega} \lambda \subseteq M$ (and therefore ${ }^{\kappa} \lambda \subseteq M$ ) and some $g:{ }^{\kappa} \lambda \rightarrow \kappa$ in $M$ satisfies $f \leq g$, then $A \in M$.

Proof. Fix $\kappa, \lambda$, and $A$. Define $f:{ }^{\kappa} \lambda \rightarrow \kappa$ by

$$
f(x):= \begin{cases}0 & \text { if }(\forall \alpha<\kappa) x(\alpha) \notin A, \\ \alpha+1 & \text { if } x(\alpha) \in A \text { but }(\forall \beta<\alpha) x(\beta) \notin A .\end{cases}
$$


This is the analogue of the function $f$ defined in Lemma 2.1. Now fix $M$ and some $g:{ }^{\kappa} \lambda \rightarrow \kappa$ in $M$ satisfying $f \leq g$. Note that $2^{\omega} \in M$ so therefore $\kappa, \lambda \in M$. Let

$$
B:=\left\{t \in{ }^{<\kappa} \lambda: g(x) \geq \operatorname{Dom}(t) \text { for all } x \text { extending } t\right\} .
$$

Since ${ }^{<\kappa} \lambda \cup\{\kappa, \lambda, g\} \subseteq M$, also $B \in M$.

Assume towards a contradiction, that $A \notin M$. Arguing just as in Lemma 2.1, there is some $x \in{ }^{\kappa} \lambda$ satisfying $(\forall \alpha<\kappa) x \uparrow \alpha \in B$. Since ${ }^{\kappa} \lambda \subseteq M$, we have $x \in M$, and in particular $x$ is in the domain of $g$. We now have $(\forall \alpha<\kappa) g(x) \geq \alpha$, which is impossible.

Lemma 4.6. Let $\kappa$ and $\lambda$ be such that $\omega \leq \kappa<\mathfrak{t}$ and $\lambda \leq 2^{\omega}$. Let $M$ be a transitive model of $Z F C$ such that ${ }^{\omega} \lambda \subseteq M$ (and therefore ${ }^{\kappa} \lambda \subseteq M$ ). Assume that for each $f:\left(\lambda^{\kappa}\right)^{M} \rightarrow \kappa$ there is some $g:\left(\lambda^{\kappa}\right)^{M} \rightarrow \kappa$ in $M$ satisfying $f \leq g$. Then $\mathcal{P}(\lambda) \subseteq M$.

Proof. This follows immediately from the previous lemma.

Now follows the theorem:

Theorem 4.7. Let $\mathbb{B}$ be a complete Boolean algebra. Let $\kappa$ and $\lambda$ be such that $1 \Vdash_{\mathbb{B}}(\check{\kappa}<\mathfrak{t})$ and $1 \Vdash_{\mathbb{B}}\left(\check{\lambda} \leq 2^{\omega}\right)$. Assume that $\mathbb{B}$ is $(\omega, \lambda)$-distributive and weakly $\left(\lambda^{\kappa}, \kappa\right)$-distributive. Then $\mathbb{B}$ is $(\lambda, 2)$ distributive.

\section{Suslin Algebras And MA $\left(\omega_{1}\right)$}

The theorems in this paper relied on absoluteness results concerning trees. We can get a counterexample to a generalization of these theorems by using a Suslin tree (a tree of height $\omega_{1}$ such that every branch and antichain is at most countable). Recall the following definition:

Definition 5.1. A Suslin algebra is a complete Boolean algebra that is atomless, $(\omega, \kappa)$-distributive for each cardinal $\kappa$, and c.c.c.

It is a theorem of ZFC that there exists a Suslin algebra iff there exists a Suslin tree. Furthermore, given a Suslin algebra $\mathbb{B}$, there is a Suslin tree (turned upside down) that completely embeds into $\mathbb{B}$, so $\mathbb{B}$ is not $\left(\omega_{1}, 2\right)$-distributive (see [6]). Immediately, we see that Theorem (B) cannot be changed by simply replacing the weakly compact cardinal $\kappa$ with $\omega_{1}$ :

Counterexample 5.2. Let $\mathbb{B}$ be a Suslin algebra. Then $\mathbb{B}$ is weakly $\left(2^{\omega_{1}}, \omega_{1}\right)$-distributive and $\mathbb{B}$ is $(\omega, 2)$-distributive, but $\mathbb{B}$ is not $\left(\omega_{1}, 2\right)$ distributive. 
Proof. The only claim left to be verified is that $\mathbb{B}$ is weakly $\left(2^{\omega_{1}}, \omega_{1}\right)$ distributive. In fact, we will show that $\mathbb{B}$ is weakly $\left(\lambda, \omega_{1}\right)$-distributive for all $\lambda$. To see why, fix $\lambda$ and fix a $\mathbb{B}$-name $\dot{f}$ such that

$$
1 \Vdash_{\mathbb{B}} \dot{f}: \check{\lambda} \rightarrow \omega_{1} \text {. }
$$

Since $\mathbb{B}$ has the c.c.c., there are only countably many possible values for a given term in the forcing language. In particular, for each $\alpha<\lambda$, there are only countably many possible values for $\dot{f}(\check{\alpha})$. For each $\alpha<\lambda$, let $g(\alpha)<\omega_{1}$ be the supremum of these possible values. We now have

$$
1 \Vdash_{\mathbb{B}}(\forall \alpha<\check{\lambda}) \dot{f}(\alpha) \leq \check{g}(\alpha) .
$$

Since $\dot{f}$ was arbitrary, by Fact $1.2 \mathbb{B}$ is weakly $\left(\lambda, \omega_{1}\right)$-distributive.

Unfortunately, the counterexample above used a Suslin algebra, which ZFC does not prove exists. In particular, we ask the following:

Question 5.3. Is it consistent with ZFC that every complete Boolean algebra that is both $(\omega, \kappa)$-distributive for all $\kappa$ and weakly $\left(\lambda, \omega_{1}\right)$ distributive for all $\lambda$ must also be $\left(\omega_{1}, 2\right)$-distributive?

The intuitive way to try to affirmatively answer the above question is to consider a model of $\mathrm{MA}\left(\omega_{1}\right)$. By Proposition 4.3, we only need to worry about those $\mathbb{B}$ such that $1 \Vdash_{\mathbb{B}}\left(\omega_{1}=\mathfrak{t}\right)$. We present another result which shows we do not need to worry about complete Boolean algebras that satisfy both a strong chain condition and enough weak distributivity laws. The main idea is the following: if we have a size $\lambda$ collection $\mathcal{C}$ of antichains in $\mathbb{B}$ each of size $\kappa^{\prime}$, then if $\mathbb{B}$ is weakly $\left(\lambda, \kappa^{\prime}\right)$-distributive, then there is a maximal antichain $A \subseteq \mathbb{B}$ such that below each $a \in A$, each antichain in $\mathcal{C}$ has $<\kappa^{\prime}$ non-zero elements. Assuming also that $\mathbb{B}$ is $(\omega,|\mathbb{B}|)$-distributive, we can repeatedly apply this construction countably many times until we produce a maximal antichain $B_{\omega}$ such that below each $b^{\prime} \in B_{\omega}$, each antichain of $\mathbb{B}$ has only countably many non-zero elements. That is, $B_{\omega}$ will witness that $\mathbb{B}$ is "locally c.c.c.". Then, we use a result of Baumgartner to conclude that since $\mathbb{B}$ is locally c.c.c. and $(\omega, 2)$-distributive, $\mathbb{B}$ is either $\left(\omega_{1}, 2\right)$ distributive or a Suslin tree can be embedded into $\mathbb{B}$. If we assume there are no Suslin trees (which follows from $\mathrm{MA}\left(\omega_{1}\right)$ ), we get that $\mathbb{B}$ must be $\left(\omega_{1}, 2\right)$-distributive.

Theorem (D). Assume there are no Suslin trees. Let $\mathbb{B}$ be a complete Boolean algebra such that $\mathbb{B}$ is $(\omega,|\mathbb{B}|)$-distributive, $\mathbb{B}$ is $\kappa$-c.c. for some $\kappa<\aleph_{\omega_{1}}$, and $\left(\forall\right.$ uncountable $\left.\kappa^{\prime}<\kappa\right) \mathbb{B}$ is weakly $\left(|\mathbb{B}|^{\kappa^{\prime}}, \kappa^{\prime}\right)$-distributive. Then $\mathbb{B}$ is $\left(\omega_{1}, 2\right)$-distributive. 
Proof. We will construct a sequence of maximal antichains

$$
\left\langle B_{n} \subseteq \mathbb{B}: n \in \omega\right\rangle
$$

such that $B_{0}:=\left\{1_{\mathbb{B}}\right\}$ and $(\forall n<m<\omega) B_{m}$ refines $B_{n}$. Each $B_{n}$ will have the property that for any maximal antichain $A$ below an element $b \in B_{n}$, for each $b^{\prime} \in B_{n+1}$ extending $b, A$ will have $<|A|$ non-zero elements below $b^{\prime}$. We will then define the maximal antichain $B_{\omega}$ to refine each $B_{n}$, and we will argue that below each $b_{\omega} \in B_{\omega}$, $\mathbb{B}$ is c.c.c.

Let $\kappa<\aleph_{\omega_{1}}$ be the least cardinal such that $\mathbb{B}$ is $\kappa$-c.c. Define $B_{0}:=$ $\left\{1_{\mathbb{B}}\right\}$. We will now define a maximal antichain $B_{1} \subseteq \mathbb{B}$ (which trivially refines $\left.B_{0}\right)$. Every antichain in $\mathbb{B}$ has size $<\kappa$. Consider an uncountable cardinal $\kappa^{\prime}=\aleph_{\alpha}<\kappa$. Let $\lambda:=|\mathbb{B}|^{\kappa^{\prime}}$. Let $\left\langle A_{\beta}: \beta<\lambda\right\rangle$ be an enumeration of the maximal antichains in $\mathbb{B}$ of size $\kappa^{\prime}$. For each $\beta<\lambda$, let $\left\langle a_{\beta, \gamma}: \gamma<\kappa^{\prime}\right\rangle$ be an enumeration of the elements of $A_{\beta}$. Let $\dot{G}$ be the canonical name for the generic filter. Fix a name $\dot{f}$ such that $1 \Vdash \dot{f}: \check{\lambda} \rightarrow \check{\kappa}^{\prime}$ and

$$
1 \Vdash(\forall \beta<\check{\lambda}) \check{a}_{\beta, \dot{f}(\beta)} \in \dot{G} .
$$

By hypothesis, $\mathbb{B}$ is weakly $\left(\lambda, \kappa^{\prime}\right)$-distributive, so there is a maximal antichain $C_{0, \alpha} \subseteq \mathbb{B}$ (which trivially refines $B_{0}$ ) and a name $\dot{g}$ such that $1 \Vdash \dot{g}: \check{\lambda} \rightarrow \check{\kappa}^{\prime}$ and

$$
1 \Vdash(\forall \beta<\check{\lambda}) \dot{f}(\beta) \leq \dot{g}(\beta)
$$

Hence,

$$
1 \Vdash(\forall \beta<\check{\lambda})\left(\forall \gamma<\check{\kappa}^{\prime}\right) \gamma>\dot{g}(\beta) \Rightarrow \check{a}_{\beta, \gamma} \notin \dot{G} .
$$

This implies that below each $c \in C_{0, \alpha}$, each $A_{\beta}$ has $<\left|A_{\beta}\right|=\kappa^{\prime}$ nonzero elements. That is, for each $c \in C_{0, \alpha}$ and $A_{\beta}$, there are $<\left|A_{\beta}\right|$ many $a \in A_{\beta}$ such that $c \wedge a \neq 0_{\mathbb{B}}$.

For each $\aleph_{\alpha}<\kappa$, we have such a maximal antichain $C_{0, \alpha} \subseteq \mathbb{B}$. Since $\kappa<\aleph_{\omega_{1}}$, the family $\left\langle C_{0, \alpha} \subseteq \mathbb{B}: \aleph_{\alpha}<\kappa\right\rangle$ is countable. Each $C_{0, \alpha}$ has size $\leq|\mathbb{B}|$, so since $\mathbb{B}$ is $(\omega,|\mathbb{B}|)$-distributive, we may fix a single maximal antichain $B_{1} \subseteq \mathbb{B}$ which refines each $C_{0, \alpha}$. Note that $B_{1}$ has the property that for each maximal antichain $A \subseteq \mathbb{B}$ (below $1_{\mathbb{B}}$ ) and $b^{\prime} \in B_{1}, A$ has $<|A|$ non-zero elements below $b^{\prime}$.

We will now define $B_{2}$. Consider an uncountable cardinal $\kappa^{\prime}=\aleph_{\alpha}<$ $\kappa$. Let $\lambda:=|\mathbb{B}|^{\kappa^{\prime}}$. Let $\left\langle A_{\beta}: \beta<\lambda\right\rangle$ be an enumeration of all size $\kappa^{\prime}$ antichains that are each a partition of some element of $B_{1}$. Since $\mathbb{B}$ is weakly $\left(\lambda, \kappa^{\prime}\right)$-distributive, we may use a similar argument as before to get a maximal antichain $C_{1, \alpha}$ which refines $B_{1}$ such that below each $c \in$ $C_{1, \alpha}$, each $A_{\beta}$ has $<\left|A_{\beta}\right|=\kappa^{\prime}$ non-zero elements. This completes the construction of $C_{1, \alpha}$. As before, we may use the $(\omega,|\mathbb{B}|)$-distributivity of $\mathbb{B}$ to get a common refinement $B_{2}$ of every maximal antichain in the 
family $\left\langle C_{1, \alpha}: \aleph_{\alpha}<\kappa\right\rangle$. Note that $B_{2}$ has the property that for every partition $A$ of some element of $B_{1}$ and $b^{\prime} \in B_{2}, A$ has $<|A|$ non-zero elements below $b^{\prime}$.

We may continue this procedure to get a sequence $\left\langle B_{n}: n \in \omega\right\rangle$ of maximal antichains of $\mathbb{B}$. The following diagram depicts the maximal antichains which we have constructed, where an arrow represents refinement:

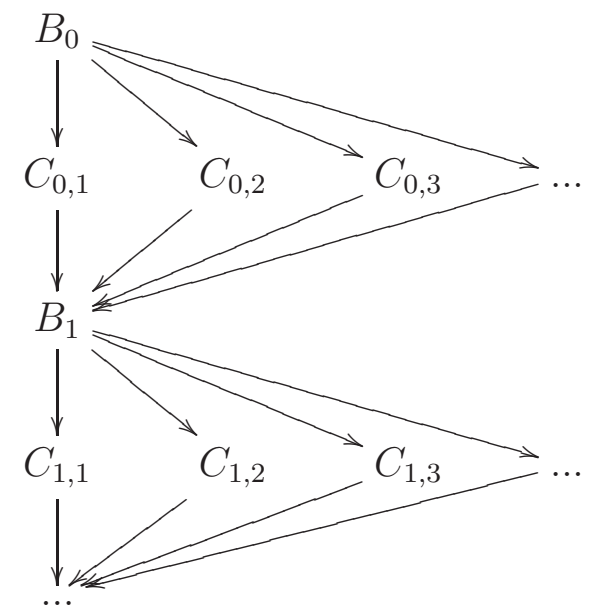

Using the $(\omega,|\mathbb{B}|)$-distributivity of $\mathbb{B}$ once more, we may get a single maximal antichain $B_{\omega} \subseteq \mathbb{B}$ which refines each $B_{n}$. We will now argue that given any maximal antichain $A \subseteq \mathbb{B}$ and $b_{\omega} \in B_{\omega}$, $A$ has only countably many non-zero elements below $b$.

Fix an arbitrary maximal antichain $A_{0} \subseteq \mathbb{B}$. Fix $b_{\omega} \in B_{\omega}$. Let $\kappa_{0}:=\left|A_{0}\right|$. If $\kappa_{0} \leq \omega$, we are done. If not, let $b_{1}$ be the unique element of $B_{1}$ above $b_{\omega}$. By the construction of $B_{1}, A_{0}$ has $<\kappa_{0}$ nonzero elements below $b_{1}$. Let $\kappa_{1}<\kappa_{0}$ be the number of such non-zero elements. That is, letting

$$
A_{1}:=\left\{a \wedge b_{1}: a \in A_{0}\right\},
$$

we have $\left|A_{1}\right|=\kappa_{1}<\kappa_{0}$. If $\kappa_{1} \leq \omega$, we are done because $\mid\left\{a \wedge b_{\omega}\right.$ : $\left.a \in A_{0}\right\}|\leq| A_{1} \mid \leq \omega$. Otherwise, let $b_{2}$ be the unique element of $B_{2}$ above $b_{\omega}$. By the construction of $B_{2}, A_{1}$ has $<\kappa_{1}$ non-zero elements below $b_{2}$. Let $\kappa_{2}<\kappa_{1}$ be the number of such non-zero elements. That is, letting

$$
A_{2}:=\left\{a \wedge b_{2}: a \in A_{1}\right\},
$$

we have $\left|A_{2}\right|=\kappa_{2}<\kappa_{1}$. If $\kappa_{2} \leq \omega$, we are done by similar reasons as before. If not, then we may continue the procedure. However, the procedure will eventually terminate. This is because if not, then we would have an infinite sequence of decreasing cardinals

$$
\kappa_{0}>\kappa_{1}>\kappa_{2}>\ldots
$$


which is impossible. Thus, $A_{0}$ has only countably many non-zero elements below $b_{\omega}$.

At this point, we have argued that below the maximal antichain $B_{\omega}$, $\mathbb{B}$ has the c.c.c. Now, it must be that $\mathbb{B}$ is $\left(\omega_{1}, 2\right)$-distributive. Let us explain. It suffices to show that $\mathbb{B}$ is $\left(\omega_{1}, 2\right)$-distributive below each element of $B_{\omega}$. Fix any $b_{\omega} \in B_{\omega}$. Below $b_{\omega}, \mathbb{B}$ is c.c.c. and $(\omega, 2)$ distributive. Suppose, towards a contradiction, that $\mathbb{B}$ is not $\left(\omega_{1}, 2\right)$ distributive. Quoting a result of Baumgartner 1 , there exists a Suslin tree which, when turned upside down, can be embedded into $\mathbb{B}$ below $b_{\omega}$. However, we assumed there are no Suslin trees. This completes the proof.

\section{REFERENCES}

[1] A. Blass. Combinatorial cardinal characteristics of the continuum. In M. Foreman and A. Kanamori, editors, Handbook of Set Theory Volume 1, pages 395489. Springer, New York, NY, 2010.

[2] P. Dordal. Towers in $[\omega]^{\omega}$ and ${ }^{\omega} \omega$. Ann. Pure Appl. Logic, 45:247-276, 1989.

[3] I. Farah. OCA and towers in $\mathrm{P}(\mathrm{N}) /$ fin. Comment. Math. Univ. Carolin., 37:861-866, 1996.

[4] D Hathaway. A lower bound for generalized dominating numbers. arXiv:1401.7948

[5] T. Jech. Distributive laws. In R. Bonnet and J.D. Monk, editors, Handbook of Boolean Algebra. North-Holland Publishing Co., Amsterdam, 1989.

[6] T. Jech. Set Theory, The Third Millennium Edition, Revised and Expanded. Springer, New York, NY, 2002.

[7] A. Kamburelis. On the weak distributibity game. Ann. Pure Appl. Logic, 66:1926, 1994.

[8] D. Martin and R. Solovay. Internal Cohen Extensions. Ann. Math. Logic, 2: 143-178, 1970.

Mathematics Department, University of Denver, Denver, CO 80208, U.S.A.

E-mail address: Daniel.Hathaway@du.edu

\footnotetext{
${ }^{1}$ This was discovered independently by Andreas Blass who was told it was already proved by James Baumgartner. However, neither the author nor Blass have been able to find a proof in the literature.
} 